\title{
¿Cuál será el costo de la salud mañana en Francia?
}

\author{
José Fabián Parra-Acosta ${ }^{1}$ \\ Diego Mauricio González Gómez² \\ Sara Dulbecco ${ }^{3}$
}

Recibido: 14 de junio de 2014

Aprobado: 17 de octubre de 2014

\section{Parra, J., González, D. \& Dulbeco, S. (2014). ¿Cuál será el costo de la salud mañana en Francia? Activos 23, 37-68}

Clasificación JEL: M14

\section{Resumen}

Este artículo presenta un análisis desde la economía de la salud, particularmente del costo futuro de la salud en Francia, tomando como eje central lo expuesto por el investigador francés Gérard Cornilleau en su artículo Quel

1 Docente de tiempo completo de la Universidad Santo Tomás; Docente ocasional de la Universidad Nacional de Colombia; director del Grupo de Estudios en Contabilidad de Costos y Control de Gestión - Control + Ge la Universidad Nacional de Colombia.Correo electrónico: jose.parra@usantotomas.edu.co,jfparraac@unal. edu.co

2 Contador público y magíster en Administración de la Universidad Nacional de Colombia; profesional de costos del Instituto para la Evaluación de la Educación ICFES. Correo electrónico: dgonzalez@icfes.gov.co,dmgonzalezg@unal.edu.co

3 Master 2: Gestion des Organisations Sanitaires et Sociales, Universite Paris XIII; Master 2: Relations Internationales, IEP Paris. Consultora de la firma P\&P Consult, París. Correo electrónico: saradulbecco@gmail.com 
sera le coût de la santé demain? Entre diversos factores, se enseñan los principales elementos y variables que se tienen en cuenta en la determinación del costo-gasto de la salud, frente a la pregunta por cómo se pueden mejorar dichos procesos de medición tan vitales en cualquier economía mundial.

\title{
Palabras clave
}

Economía de la salud, costo de la salud, gasto en salud, salud en Francia.

Parra, J., González, D. \& Dulbeco, S. (2014). What will be the cost of health tomorrow in France?. Activos 23, 37-68

\begin{abstract}
This paper presents an analysis from the health economics, particularly the future cost of health in France, taking as central theme what is stated by French researcher Gérard Cornilleau in his article Quel sera le coût de la santé demain? Among several factors, the main elements and variables that are taken into account in determining the cost-expenditure of health are shown, with regards to the question of how to improve these measurement processes so vital in any world economy.
\end{abstract}

\section{Keywords}

Health economics, health costs, health expenditure, health in France. 


\section{Parra, J., González, D. \& Dulbeco, S. (2014). Quel sera le coût de la santé demain en France?. Activos 23, 37-68}

\section{Résumé}

Cet article présente une analyse à partir de l'économie de la santé, et particulièrement du coût futur de la santé en France, en prenant comme point central l'argument présenté para le chercheur français Gérard Cornilleau dans son article Quel sera le coût de la santé demain? Parmi différents facteurs, on révèle les principaux éléments et variables qui sont pris en compte dans la fixation de la variable coût-dépense de la santé, face à la question de comment il est possible d améliorer ces processus de mesure aussi vitaux dans $n$ 'importe quelle économie mondiale.

\section{Mots clés}

Économie de la santé, coût de la santé, dépense de santé, santé en France. 


\section{Introducción}

Al terminar la Segunda Guerra Mundial, en Francia nace el sistema de protección social, bajo la idea de una seguridad social para todos. El 4 de octubre de 1945 una ordenanza de Pierre Laroque, en ese entonces ministro de trabajo, da origen a la Caja Nacional del Régimen de Seguridad General de la Seguridad Social, definida como

"Una organización destinada a garantizar a los trabajadores y sus familias la protección necesaria contra riesgos de toda naturaleza... así como de cubrir los gastos de maternidad y otros gastos familiares" (L'Assurance Maladie, 2008, p. 1).

Aparece por primera vez en el preámbulo de la Constitución en 1946 el derecho a la seguridad social, según el cual

La Nación asegura al individuo y a su familia las condiciones necesarias para su desarrollo. Ella (la seguridad social) garantiza a todos, en especial a los niños, madres y trabajadores mayores, la protección de la salud, de la seguridad material, el descanso y el entretenimiento [...]. (Asamblée National - République Française, 1946, pp. 10-11)

Si bien el sistema de protección social ha sido susceptible de varias reformas desde su creación, la cobertura de los riesgos de salud permanece como una problemática central en esta transformación. Aunque la salud no puede considerarse un costo, sí tiene un valor que crece proporcionalmente con las evoluciones estructurales del entorno (demografía, economía, estado del sistema de salud, entre otros).

Los gastos en salud presentan un carácter específico por su indiscutible necesidad, para los enfermos y para la población en general. Los factores exógenos que impactan su evolución son varios, mientras que los factores que regulan e influencian el consumo "clásico" (aquellos relacionados con la atención curativa directa), o tienen la misma incidencia sobre aquellos. La salud es un mercado específico en el sentido de que es una actividad no 
programable, sujeta a la reglamentación de los precios, los subsidios del Estado y la asimetría de información.

La economía es la ciencia que tiene por objeto la producción, distribución y consumo de bienes y servicios. Aplicada a la salud, esta disciplina cubre el campo de todos los bienes y servicios destinados a procurar "el estado completo de bienestar físico, social y mental, y no solamente la ausencia de enfermedad"(Kinnon, Velazquez, \& Flori, 1994). En este sentido, el objetivo de la economía de la salud es estudiar las restricciones del entorno socioeconómico, con el objetivo de optimizar la utilización de los recursos financieros, materiales, técnicos y humanos en función de este entorno.

El sistema de salud francés funciona de manera asegurativa y asistencial; si bien no permite a los usuarios del sistema conocer el costo exacto de los servicios que utilizan, es una gran herramienta financiera porque los demanda por su capacidad de financiamiento inmediato de salud, es decir, la disponibilidad inmediata de recursos económicos que permitan el pago de la atención médica. Sin embargo, un costo real existe y está respaldado directa e indirectamente a través de:

- la seguridad social básica que comprende el déficit en los hospitales públicos;

- el Estado y la cobertura universal de enfermedades;

- los organismos complementarios:

- las cooperativas,

- las compañías de seguros e

- instituciones de previsión;

- los hogares. 
En 2011 el presupuesto destinado para el consumo de tratamientos y productos médicos (Consommation de Soin et de Biens Médicaux [CSBM]) se estimó en 186,7 mil millones de euros, cantidad que representa unos 2843 euros por habitante. Así, el CSBM equivalió al 8,8 \% del PIB en 2011, y el 8,7\% en 2012. El gasto corriente en salud representó el 11,6\% del PIB en 2012, el 11,5\% en 2011, y en 2013 se elevó a 247,7 mil millones de euros (11,7\% del PIB).

Para analizar el valor del gasto en salud en la sociedad existen dos modelos. El primero consiste en llevar el valor actual de consumo y el producto interno bruto (PIB). El segundo se centra en los cambios en el volumen de consumo. La gestión del gasto en salud es una de las prioridades de la seguridad social, ya que en los últimos años los fondos de seguros de salud enfrentan un fuerte aumento en el volumen y la diversidad de prestaciones. Al mismo tiempo, no existe un método universal de aproximación a la problemática.

El artículo escogido como insumo principal para la discusión de este documento se titula: Quel sera le coût de la santé demain? (¿Cuál será el costo de la salud mañana?'), escrito por el investigador y economista francés Gérard Cornilleau (2009). El objetivo es determinar la dirección de la evolución de los gastos en salud a largo plazo, y especificar los factores determinantes con base en las observaciones anteriores.

Inicialmente se analizan las causas de un crecimiento en gastos en salud más rápido que el del PIB. Después se determina el impacto de la actual crisis económica y el envejecimiento de la población, dos indicadores contemporáneos que, junto con otros elementos macroeconómicos y macro sociales que impulsarán la evolución de los gastos en salud, explican este crecimiento. 


\section{Factores y mecanismos de determinación del costo de la salud}

En esta sección se discuten los factores y mecanismos de determinación del costo de la salud. Se realiza una extrapolación de las tendencias pasadas, seguida de una presentación y análisis de las variables macroeconómicas y sociales utilizadas en el presente documento como factores explicativos del aumento continuo del gasto en salud.

\section{A. La extrapolación de los gastos anteriores}

La salud no es un costo. Los gastos relacionados con ella constituyen un consumo particular del ingreso que contribuye, junto con el consumo de otros recursos, al bienestar de la población. El costo del servicio de salud es insensible a las variables habituales que determinan el consumo privado: el ingreso del consumidor y el precio relativo del producto o servicio solicitado.

Los factores que determinan la evolución del gasto en salud son en gran medida exógenos, y los mecanismos habituales de autorregulación (restricciones presupuestarias individuales y precio) juegan un papel menor. La proyección del gasto en salud se basa principalmente en la extrapolación de tendencias pasadas.

\section{i. Elasticidad y PIB}

El gasto en salud creció más rápido que el PIB en todos los países de la Organización para la Cooperación y el Desarrollo Económicos (OCDE). la tendencia de 2010 muestran que la tendencia es igual para Francia (Figura 1). 
Figura 1. Gasto total en salud en Francia/ PIB

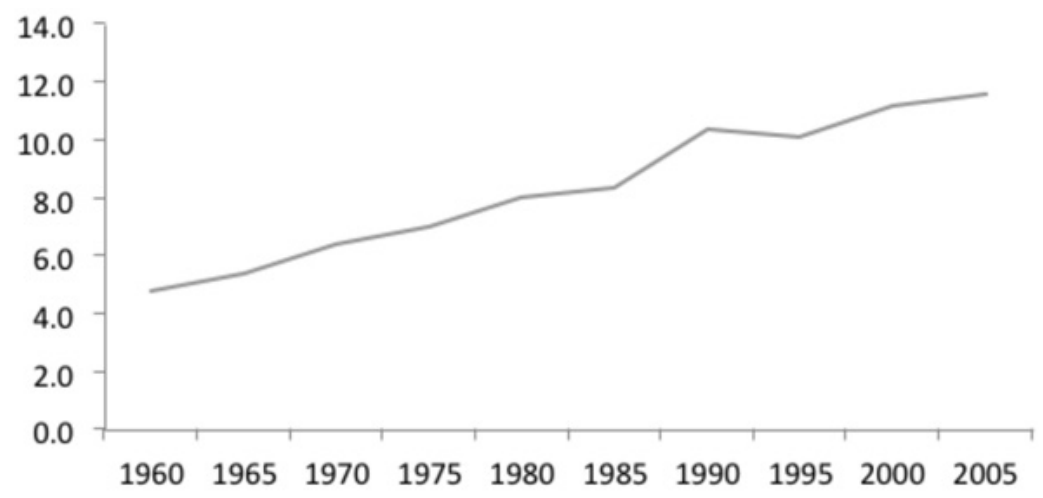

Fuente: elaboración propia a partir de la OCDE (2012).

Tabla 1. Porcentaje del gasto en salud en el PIB

\begin{tabular}{l|c|c|c|c|c|c|c|c|c}
\hline \multirow{2}{*}{\multicolumn{1}{c|}{ País }} & \multicolumn{5}{|c|}{ Año } & \multicolumn{5}{c}{ Variaciones } \\
\cline { 2 - 11 } & 1975 & 1985 & 1995 & 2005 & 2010 & $\begin{array}{c}1975- \\
1985\end{array}$ & $\begin{array}{c}1985- \\
1995\end{array}$ & $\begin{array}{c}1995- \\
2005\end{array}$ & $\begin{array}{c}2005- \\
2010\end{array}$ \\
\hline Austria & 7,0 & 6,5 & 9,8 & 10,2 & 11,0 & $(0,5)$ & 3,3 & 0,4 & 0,8 \\
\hline Bélgica & 5,6 & 7,0 & 8,2 & 10,3 & 10,5 & 1,4 & 1,2 & 2,1 & 0,2 \\
\hline $\begin{array}{l}\text { República } \\
\text { Checa }\end{array}$ & - & - & 7,0 & 7,2 & 7,5 & - & 7,0 & 0,2 & 0,3 \\
\hline Dinamarca & 8,7 & 8,5 & 8,1 & 9,1 & 11,1 & $(0,2)$ & $(0,4)$ & 1,0 & 2,0 \\
\hline Finlandia & 6,2 & 7,1 & 7,5 & 7,5 & 8,9 & 0,9 & 0,4 & - & 1,4 \\
\hline Francia & 6,4 & 8,0 & 9,9 & 11,1 & 11,6 & 1,6 & 1,9 & 1,2 & 0,5 \\
\hline Alemania & 8,4 & 8,8 & 10,1 & 10,7 & 11,6 & 0,4 & 1,3 & 0,6 & 0,9 \\
\hline Grecia & - & - & 7,5 & 10,1 & 10,2 & - & 7,5 & 2,6 & 0,1 \\
\hline Hungría & - & - & 7,3 & 8,4 & 7,8 & - & 7,3 & 1,1 & $(0,6)$ \\
\hline Irlanda & 7,3 & 7,5 & 6,7 & 7,5 & 9,2 & 0,2 & $(0,8)$ & 0,8 & 1,7 \\
\hline Italia & - & - & 7,3 & 8,9 & 9,3 & - & 7,3 & 1,6 & 0,4 \\
\hline Japón & 5,7 & 6,7 & 6,9 & - & 9,5 & 1,0 & 0,2 & $(6,9)$ & 9,5 \\
\hline Luxemburgo & 4,3 & 5,2 & 5,6 & 7,9 & 7,9 & 0,9 & 0,4 & 2,3 & - \\
\hline
\end{tabular}




\begin{tabular}{l|c|c|c|c|c|c|c|c|c}
\hline \multirow{2}{*}{\multicolumn{1}{c|}{ País }} & \multicolumn{5}{|c|}{ Año } & \multicolumn{5}{c}{ Variaciones } \\
\cline { 2 - 11 } & 1975 & 1985 & 1995 & 2005 & 2010 & $\begin{array}{c}1975- \\
1985\end{array}$ & $\begin{array}{c}1985- \\
1995\end{array}$ & $\begin{array}{c}1995- \\
2005\end{array}$ & $\begin{array}{c}2005- \\
2010\end{array}$ \\
\hline Países Bajos & 7,1 & 7,3 & 8,3 & - & 12,0 & 0,2 & 1,0 & $(8,3)$ & 12,0 \\
\hline Polonia & - & - & 5,5 & 6,2 & 7,0 & - & 5,5 & 0,7 & 0,8 \\
\hline Portugal & 5,1 & 5,7 & 7,8 & 10,2 & 10,7 & 0,6 & 2,1 & 2,4 & 0,5 \\
\hline $\begin{array}{l}\text { República } \\
\text { Eslovaca }\end{array}$ & - & - & - & 7,1 & 9,0 & - & - & 7,1 & 1,9 \\
\hline España & 4,6 & 5,4 & 7,4 & 8,3 & 9,6 & 0,8 & 2,0 & 0,9 & 1,3 \\
\hline Suecia & 7,6 & 8,6 & 8,1 & 9,1 & 9,6 & 1,0 & $(0,5)$ & 1,0 & 0,5 \\
\hline Suiza & 7,0 & 7,8 & 9,7 & 11,6 & 11,4 & 0,8 & 1,9 & 1,9 & $(0,2)$ \\
\hline Reino Unido & 5,5 & 5,9 & 7,0 & 8,3 & 9,6 & 0,4 & 1,1 & 1,3 & 1,3 \\
\hline $\begin{array}{l}\text { Estados } \\
\text { Unidos }\end{array}$ & 7,9 & 10,0 & 13,3 & 15,3 & 17,6 & 2,1 & 3,3 & 2,0 & 2,3 \\
\hline
\end{tabular}

Fuente: OCDE (2012).

Figura 2. Gasto en salud, público y privado, como proporción del PIB, en los países de la OCDE, 2010

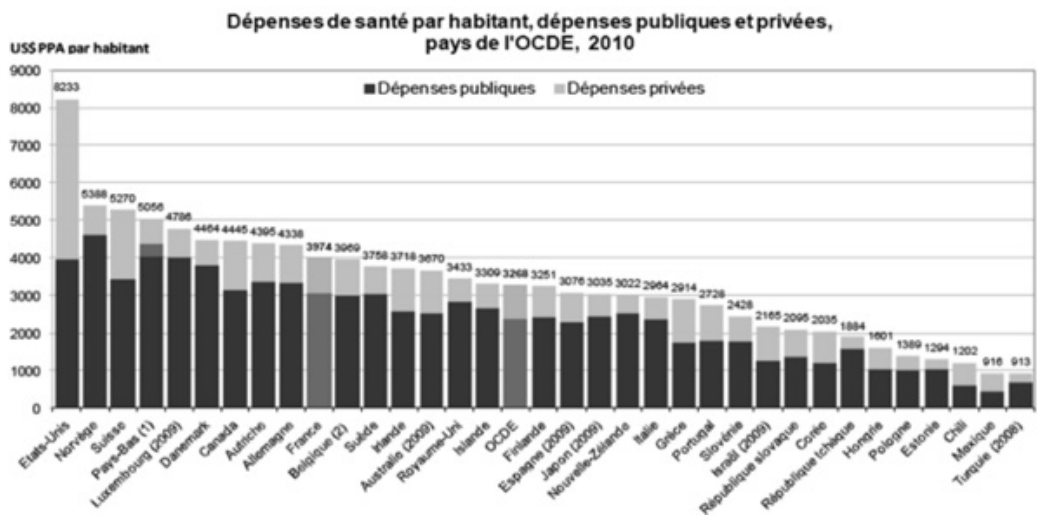

Fuente: OCDE (2012). 
Figura 3. Gasto público en salud con respecto al gasto total en salud en Francia (en porcentaje)

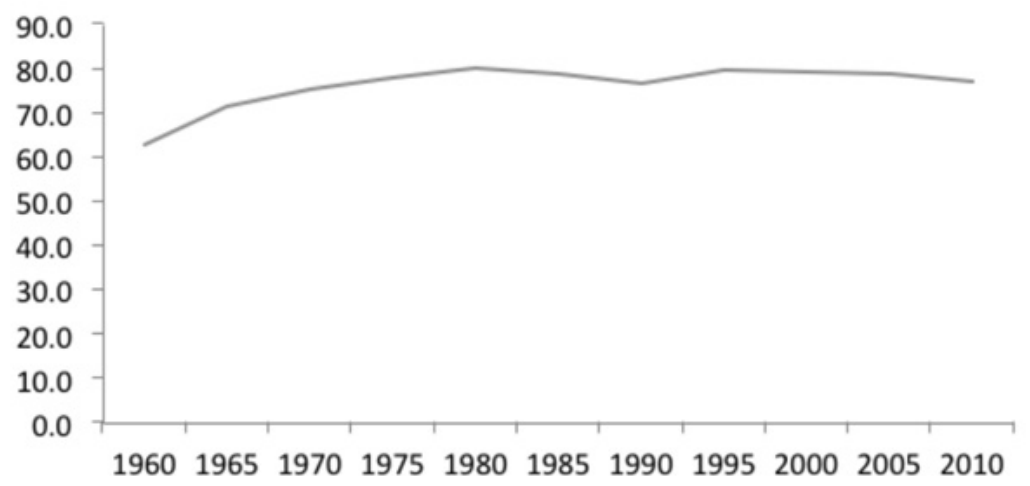

Fuente: elaboración propia con base en OCDE (2012).

Este crecimiento en el gasto destaca el problema de la financiación a largo plazo: "En un contexto de crecimiento y financiación socializada del gasto en salud, la cuestión se posa en el nivel deseable de gasto"(Fargeon, 2009, p. 42).

La evolución de la financiación depende de la economía del país, mientras que los gastos de salud dependen de otras variables: el nivel de vida, el envejecimiento y los avances en las técnicas médicas. La disociación de variables explica, en parte, el origen de los desequilibrios de la financiación del sistema de salud francés. El fallo progresivo de la financiación en relación con el gasto de salud requiere una respuesta política.

...dado que peligra el financiamiento, se requiere una respuesta urgente del gobierno. En general, un paquete de medidas pretende el no reembolso de algunas prestaciones y el aumento de cuotas. Cuando el crecimiento es suplido, la situación es ligeramente más favorable. El financiamiento del seguro médico es obviamente cubierto, como fue el caso en la segunda mitad de la década de 1990, pero la situación sigue siendo problemática. (Benamouzig \& Launois, 1999, p. 4) 


\section{ii. Incertidumbre en la medición de los precios de la salud}

Para predecir el comportamiento de una variable es necesario hacer un análisis econométrico, el cual permitirá establecer los condicionamientos que la afectan. El análisis econométrico se basa en tendencias históricas de las variables explicativas; en otras palabras, si una variable se entiende por el comportamiento de otras variables, esas tendencias pueden ayudar a establecer la tendencia de la variable objeto de estudio.

Se necesita la proyección del gasto en salud para establecer políticas públicas, pero ¿cómo podemos medir con precisión los costos si estos dependen de diversas variables difíciles de establecer? Esta pregunta se presenta en el caso de los precios: "Otro factor que explica el gasto en salud es el costo de la atención. A nivel individual, donde existen mecanismos de socialización de seguro de salud, el paciente no se expone al precio total de la atención médica" (Fargeon, 2009, p. 44). Entonces, "La influencia del nivel de apoyo público o el gasto en salud socializado sigue siendo incierto" (Fargeon, 2009, p. 45).

Las comparaciones del gasto en salud a escala internacional son inciertas, debido a la incertidumbre sobre la medida de precios del gasto en salud en los diferentes países. Por lo tanto, cuando un país está experimentando un aumento en el volumen de la atención por debajo de la media, a su vez experimenta un aumento de precios de los servicios sanitarios mucho más alto.

La tasa de renovación de un medicamento también afecta los precios, efecto que puede explicarse como una alteración sobre el índice de precios de consumo (IPC). El aumento de precios de la atención de salud, debido a la introducción de un nuevo medicamento, puede causar una disminución de la demanda; sin embargo, como la tasa de sustitución no es demasiado alta, se observa una mejora de la calidad de la salud y, por lo tanto, la demanda sigue siendo casi la misma. "La subida de precios puede alentar a los proveedores de atención de salud para producir más, pero también 
puede promover el arbitraje a favor de la porción del ingreso dedicada al ocio" (Fargeon, 2009, pp. 45-46)

Hay otros factores determinantes del comportamiento del gasto en salud que han aparecido en los últimos años, tales como las atenciones ambulatorias (las enfermeras, las pruebas de laboratorio, entre otros) y los medicamentos (Fargeon, 2009).

Las dificultades estadísticas respecto a la medida del precio y el volumen de consumo de los bienes y servicios de salud, según Cornilleau, son:

- Una parte importante de la atención de salud se paga con una tarifa fija, los hospitales son financiados por un presupuesto global, y no hay un precio de mercado del servicio prestado (estimación indirecta).

- La evaluación de los precios "al costo de los factores de la producción", debido a la falta de un mercado desarrollado que arroje un precio corriente.

- La medición de los gastos reales de la atención depende en gran parte de las evaluaciones del volumen de medicamentos que se consumen en cada país.

El problema con la información estadística es que la fuente está administrada solo por el gobierno, y la información puede estar sesgada. En otras palabras, puede estar al servicio de la imagen del gobierno y presentar datos que demuestren una buena gestión de los recursos públicos.

\section{B. Influencia de las variables económicas sociales y macro}

En este aparte se habla de los determinantes del crecimiento del gasto en salud. Hay dos métodos principales para analizar los factores (variables) del crecimiento de los gastos de salud, permitiendo de esta forma extrapolar las tendencias. Antes de presentar en detalle las variables que considera 
Cornilleau, conviene presentar los dos tipos de métodos. El primero no es el utilizado por el autor, pero sí por el Haut Conseil pour l'avenir de l'assurance maladie (Consejo Superior para el Futuro del Seguro de Salud). Este método razona en función del valor corriente del consumo con relación al PIB.

El consumo en valor corresponde a los gastos realizados en un momento dado. Por lo tanto, un gasto en valor es un gasto en moneda corriente. Este método tiene en cuenta los gastos de salud con relación al PIB (esta es una relación que permite deducir un porcentaje, a modo de informe).

La ventaja de este método es que permite extrapolar una tendencia en el gasto en salud, aunque los economistas opinan que para reconocer una tendencia más precisa y basada en las tendencias en los últimos años, lo mejor es comparar el gasto en volumen. El consumo de volumen permite conocer la cantidad de bienes y servicios consumidos en moneda constante de un año de referencia, suprimiendo así el efecto de la inflación.

Aunque este método ofrece la posibilidad de conocer las tendencias, no permite analizar los factores de crecimiento. Por tales razones el autor eligió el segundo método - el método empírico- para evaluar el crecimiento del gasto en salud.

El método empírico conserva el análisis directo de los cambios en el volumen de consumo. La premisa es tener siempre en cuenta la evolución del precio de la salud, manteniendo constantes las convenciones contables. El método empírico permite retener una serie de factores explicativos endógenos en la evolución del gasto en salud. El hecho de que ningún método sea percibido como óptimo hace que sea difícil medir el gasto en salud. A continuación, el análisis se centra en las tres variables más importantes utilizadas por el autor.

\section{i. Demografía y envejecimiento}

Como se ha visto, Cornilleau, utilizando el segundo método para determinar los factores de crecimiento en el gasto en salud, revela tres variables 
principales. La primera es la demografía y el envejecimiento. Las características demográficas están intrínsecamente vinculadas. Como expresa Valerie Fargeon (2009), "El factor demográfico es un factor determinante de la demanda de atención de la salud ya que el consumo depende del tamaño de la población y no es uniforme en toda la vida”.

Para Cornilleau (2009) la variable demografía/envejecimiento es la que mejor explica todo. Es adecuada para medir el impacto del gasto en salud. De hecho, no se usa de la misma manera en función del grupo de edad al que se pertenece.

Si bien está claro que el envejecimiento de la población influye en el gasto en salud este mecanismo hay que evaluarlo, ya que deja de lado otros factores. Para ilustrar esta relación, Dormont (2011) concluye que los gastos en salud aumentan con edad de las personas -el incremento se acentúa a partir de los 50 años- (gráfica 4), de igual manera las proyecciones de envejecimiento de la población, indican que la proporción de mayores de 65 años representó el 16,1 \% de la población total en 2000, el cual se estima llegará en 2050 al 27,1\% de la población total. Sin embargo, éste análisis no tiene en cuenta el cambio en el tiempo del perfil de gastos por la edad, la figura 5 compara el gasto anual de salud por edad para 1992 y 2000 y en ella se evidencia un aumento de los patrones de consumo de bienes relacionados con la salud, a medida que aumenta la edad del individuo.

La figura 5 muestra que los patrones de consumo se están moviendo hacia arriba. De hecho, para todos los grupos de edad la proporción del gasto es mayor en 1992 que en 2000, lo que demuestra que este incremento en el gasto se da en virtud del envejecimiento de la población.

Se observa que entre 1992 y 2000 el gasto en salud por grupo de edad aumentó más del 50 \%, mientras que la proporción de mayores de 65 años, en 1992, representó solo el 14,5\% de la población, y en 2000, el 16,1\%, lo que corresponde a 1,6 puntos de aumento. Por consiguiente, algunos fenómenos podrían explicar la tendencia al alza de los perfiles de gasto por edad: 
Figura 4. Gasto anual en salud por rango de edad en 2000

Gasto Anual en Salud por rango de edad 1992 y 2000

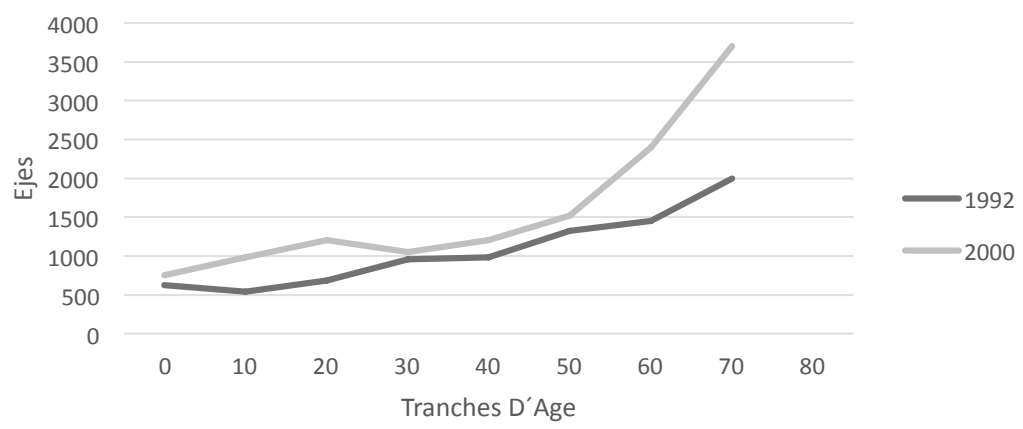

Fuente: Dormont (2011).

Figura 5. Gasto anual en salud por rango de edad para 1992 y 2000

Gasto Anual en Salud por rango de edad 1992 y 2000

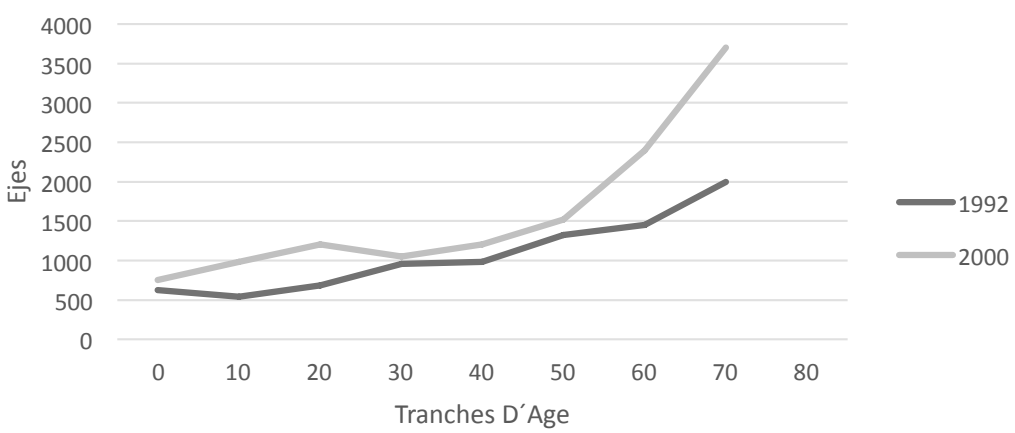

Fuente: Dormont (2011)

ISSN: 0124-5805, Revista Activos, N. ${ }^{2}$ 23, julio-diciembre de 2014, pp. 37-68 
1. Un aumento en la morbilidad.

2. El segundo sería dado a la edad y la morbilidad, las prácticas médicas mutan y eso las lleva a gastos más importantes (un individuo víctima de un ataque al corazón se realizaría más pruebas y procedimientos más costosos en 1992 que en 2000).

3. El aumento de la esperanza de vida, que viene acompañado de un mejor estado de salud (tesis que alimentaría la hipótesis de Brigitte Dormont, relacionada con los avances en tecnología médica.

Sin embargo, Cornilleau (2009) y Dormont (2009) aceptan que el impacto del envejecimiento en el aumento del gasto en salud sigue siendo moderado. La DRESS-Direction de la recherche, des études, de lévaluation et des statistiques (Dirección de la Investigación, Estudios y Evaluación de las Estadísticas) indica que la proporción del gasto en salud debido al envejecimiento es de $0,2 \%$ por año en el Reino Unido; de $0,9 \%$ por año en Italia; y de 0,8\% por año en Francia. Los trabajos de los prospectivistas establecen una estabilización de $0,73 \%$ por año durante el periodo $2010-2020$ (Direction de la Recherche des Études de l'Évaluation et des Statistiques [DREES], 2012).

Como se ha visto, el envejecimiento tiene un impacto limitado en el aumento del gasto en salud, por lo que podría pensarse en la posibilidad de explicar el fenómeno a través de la variable demográfica. Sin embargo, una desaceleración del crecimiento de la población, observada desde la década de los sesenta, que normalmente conllevaría a una disminución en el crecimiento del gasto, deja sin piso dicha hipótesis. De hecho, el gasto anual de salud por edad sigue una curva en forma de $\mathrm{U}$ (muchos gastos durante los primeros y los últimos años de vida).

De acuerdo con Cornilleau (2009), el impacto de todos los grupos demográficos es increíblemente estable desde los años sesenta, ya que es del orden del 0,8 al $1 \%$ anual. Los trabajos de prospectiva prevén una estabilización del $1 \%$ para el período 2010-2020. 
Es importante recordar aquí, en términos de crecimiento en el gasto en salud, la diferencia entre el impacto del crecimiento de la población y el impacto del envejecimiento de la población sobre la financiación del seguro de salud. Mientras que el envejecimiento implica un aumento de los costos sin incrementar el crecimiento de los ingresos, el aumento de la población significa más gastos al principio, pero más ingresos a largo plazo.

En conclusión, el autor afirma que "la contribución de las variables demográficas en el crecimiento del gasto en salud a precios constantes es de alrededor de $1 \%$ por año. Así que a comportamientos invariables, el volumen de gasto aumenta cada año, al menos un $1 \%$ ” (2009) -traducción de los autores-.

\section{ii. Aumento del nivel de vida}

Para Gérard Cornilleau, y para otro buen número de autores como Fargeon (2009) y Phelps (1995), el crecimiento de la renta per cápita es el principal promotor del aumento del gasto en salud. Por lo tanto, el aumento del nivel de vida es la segunda variable que pesa sobre el crecimiento del gasto sanitario. Las comparaciones internacionales consideran la relación entre el gasto y la riqueza nacional un factor clave. Lo que se estudia con esta variable es la naturaleza de la relación entre los ingresos y los gastos en salud.

Para Gérard Cornilleau, la demanda de los hogares aumenta con los ingresos y la oferta de atenciones médicas aumenta para satisfacer esta demanda. Sin embargo, esta relación de causalidad puede ser cuestionada, dado que los hogares tienen un conocimiento aproximado de sus necesidades en materia de salud.

Si bien el crecimiento del gasto en salud se debe en gran parte al crecimiento de la renta per cápita, surge la pregunta por el origen del gasto. Aparece así el papel del prescriptor.

Así, en las políticas de gestión de gastos en salud han surgido planes relacionados no solo con la regulación de la demanda de drogas (frenar el 
consumo a través de tarifas a los usuarios y la política del no reembolso), sino también con la gestión de la oferta a través de la introducción de los medicamentos genéricos y la conformación de las buenas prácticas para prohibir la conducta abusiva en términos de prescripción.

El punto importante aquí es que el consumo aumenta con el nivel de riqueza nacional, por lo cual se tiene una relación directamente proporcional entre el crecimiento del gasto en salud y la riqueza nacional.

Según estudios econométricos basados en datos históricos anteriores, los gastos en salud tienen una alta elasticidad en relación con el PIB, generalmente mayor a uno. En otras palabras, el aumento de la renta nacional es directamente proporcional al incremento en los costos de la salud. Para algunos autores, esto demostraría dos fenómenos: primero, la medicalización de la sociedad y segundo, una aspiración colectiva de bienestar.

Respecto a la elasticidad del gasto en salud con el PIB, que en general es mayor a uno, en promedio, un aumento del $1 \%$ en el PIB per cápita se traduciría en un aumento de más del $1 \%$ del consumo de asistencia en salud por persona.

La elasticidad es el efecto de la variación relativa en una variable en el cambio relativo en la otra variable. Durante mucho tiempo se produjo un incremento del $1 \%$ del PIB, junto con un aumento de los costos de salud, también del $1 \%$. Pero desde los años sesenta dicha coincidencia dejó de presentarse. Los cambios en el gasto en salud presentaron una elasticidad mayor a uno, lo que se tradujo en un aumento más rápido de estos con respecto al PIB.

Así, de 1960 a 1975 se tuvo una elasticidad de 2,2; para el periodo 19761990, de 1,5; y desde 1990, entre 1,2 y 1. Esta baja en el nivel de elasticidad se explica, en parte, por la introducción de los primeros planes para limitar el gasto en salud. El primero de ellos fue el plan Durafour de 1975. 
Las obras de Newhouse, en 1977, sobre trece países muestran que la variable ingresos/PIB per cápita es la más significativa para explicar la variación en el gasto en salud, ya que explicaría el $92 \%$. Según Newhouse (1977), la salud es un bien superior y la compra de una unidad de cuidados mejora el estado subjetivo de bienestar (cuidado), mucho más que los indicadores de morbilidad y mortalidad (cura).

Estudios de Murillo, Piatecki y Saez (1993) muestran que la elasticidad de ingreso -es menor a medida que el país es más rico. Esto se explica por el hecho de que el aumento del PIB per cápita permite un aumento de la base de cotización que proporciona fondos para beneficiar los gastos de salud. También se debe evaluar el enfoque de Newhouse, que elimina los efectos del progreso técnico. Así que para Cornilleau el aumento de la calidad de vida no es incompatible con la estabilización del gasto en salud.

\section{iii. Los avances en la tecnología médica}

Según Cornilleau, el supuesto de elasticidad unitaria del gasto sanitario y el aumento del PIB deben explicar por si solas, el aumento continuo del gasto en salud. Así, la explicación que se utiliza a menudo está en la naturaleza del progreso técnico, mientras que en la mayoría de las industrias el progreso técnico es sinónimo de ganancias por productividad, en el sector de la salud el efecto de dicho progreso es paradójico pues conduce a un aumento acelerado de los costos de prestación del servicio.

Antes de seguir adelante, nos detenemos en las contribuciones de los avances en la tecnología médica. Estos avances implican dos áreas: el campo del diagnóstico por imagen (MRI, CT) y el campo del diagnóstico en la terapia (trasplantes, terapia del cáncer, el desarrollo de técnicas no invasivas o drogas).

El progreso técnico podría reducir ligeramente el costo de los tratamientos existentes, pero los nuevos son siempre más caros. La ambivalencia de 
los avances en la tecnología médica es entonces saber si son generadores de ahorros o de costos.

Aunque todo el mundo está de acuerdo en que el progreso permite una mejora en la calidad de la atención, la efectividad del tratamiento y el confort, aún queda por evaluar estas mejoras desde el punto de vista contable, sin sobrevalorar o subestimar su valor.

Varios autores plantean que los avances en la tecnología médica son un factor de inflación real de los gastos en salud, y que, por ende, podrían explicar lo que otras variables no. Horthy, Quinet y Rupprecht (1997) demuestran que si bien es difícil medir el impacto de los avances técnicos, a pesar de la utilización de los indicadores directos del desarrollo de nuevas técnicas, es posible considerar que el impacto del progreso técnico es una cuarta parte de los gastos de salud en Francia.

Como dice Cornilleau, la determinación de los indicadores adecuados sigue siendo muy difícil y la extrapolación aún más. Es importante hacer referencia a los informes de expertos para evaluar el impacto futuro del progreso técnico, como el escrito por un comité estratégico, que presenta las áreas de salud susceptibles de experimentar grandes avances (médico genética, cirugía, análisis exploratorios, medicina, telemedicina, entre otros).

También es importante tener en cuenta que el progreso técnico es un proceso no lineal, que involucra dos mecanismos. Según Dormont (2009), el primer mecanismo es la sustitución del tratamiento, que permite un incremento de la eficacia y la creciente difusión del tratamiento, que corresponde a un uso cada vez mayor de la innovación. El segundo mecanismo es, para Cutler y McClellan (2001), el aumento del costo de los tratamientos, que resulta exclusivamente de la difusión de las innovaciones, porque la sustitución mejora la calidad de la atención y es la fuente de las ganancias de productividad.

Como se vio en este aparte, el progreso técnico no permite identificar fácilmente las evoluciones que generan ahorros o costos. 


\section{Prospectiva de los gastos y del costo de la salud}

En esta sección se discuten las tendencias futuras en el gasto en salud y, finalmente, las críticas y perspectivas que se pueden hacer al artículo.

\section{A. La futura evolución del gasto en salud}

El porcentaje del ingreso nacional que se destina a la salud está creciendo rápidamente desde hace décadas, por lo que es esencial evaluar la probabilidad de que esta tendencia continúe, sus causas fundamentales, y la posible influencia de las políticas públicas en el fenómeno descrito. Para ello, es necesario revisar los posibles determinantes del aumento del gasto en salud. Si bien algunos de los factores anteriores pueden tener menor relevancia en el futuro de la salud, esta sigue siendo fundamentalmente una industria en crecimiento, impulsado por la innovación técnica en el campo de la medicina.

Las estimaciones anteriores, revisadas en los documentos de la Dirección General del Tesoro y Política Económica (DGTPE), anticipan un aumento de los costos en salud en el PIB, entre 0 y más de 10 puntos, para 2050. De acuerdo con estas estimaciones de DGTPE, el impacto del envejecimiento sobre el gasto sanitario debe estar entre $1 / 2$ y $2 \frac{1}{1 / 2}$ puntos porcentuales del PIB en 2050, puesto que la salud de los adultos mejorará de forma espontánea 0 , al contrario, las atenciones médicas se intensificarán.

\section{La evolución futura del gasto en salud}

Según Gérard Cornilleau (2009) "el financiamiento por el CSG ha roto el vínculo entre los costos de mano de obra y el costo de la salud". La contribución social generalizada (CSG) es un impuesto creado para ayudar a financiar la protección social (el seguro de salud, las prestaciones familiares, las pensiones a través del Fondo de Solidaridad de la Vejez [FSV], la pérdida de autonomía a través de la Caja Nacional de Solidaridad para la Autonomía 
[CNSA] y el reembolso de la deuda social) y se aplica a todos los ingresos, a excepción de las prestaciones familiares.

Desde diciembre de 1990 se paga por toda persona domiciliada en Francia para el establecimiento del impuesto sobre la renta y, además, en cualquier condición, de un régimen obligatorio francés de un seguro de enfermedad.

La financiación de la salud de los usuarios tiene dos niveles: el trabajo (CSG) y el consumo. Esta relación pone de manifiesto la relación que existe entre el trabajo y la financiación de los costos de salud, y ahí radica la idea clave del autor.

Anteriormente, en parte financiado por las contribuciones pagadas por los empleadores, un fuerte aumento en el gasto en salud habría tenido un papel perverso sobre el empleo, aumentando los gastos de los empleadores. La contribución social generalizada permite enfrentar estas repercusiones negativas que la situación anterior habría generado.

La CGS es una reforma estructural de ayudas orientadas a mitigar los riesgos relacionados con la salud. El desarrollo de un sistema de gestión para el gasto en salud es realmente complejo y depende de la naturaleza técnica de las políticas públicas.

\section{Variables en movimiento}

\section{Demografía y envejecimiento de la población}

Entre los factores que contribuyen al dinamismo de los gastos en salud, el envejecimiento de la población parece el más predecible. En comparación con otros factores que entran a jugar un papel importante (las innovaciones médicas, la densidad y la distribución de la oferta), los cambios demográficos en Francia son relativamente bien conocidos. Aunque existe incertidumbre sobre la evolución de la fecundidad y la inmigración, se 
sabe que la combinación de un proceso de envejecimiento acelerado de la generación del "baby boom" (1950-1970) y el aumento de la expectativa de vida, son factores que demandarán una mayor relevancia del gasto sanitario.

El envejecimiento tiene un lugar central en el debate público sobre la evolución del gasto en atención de salud, tanto por su tamaño como por su previsibilidad. A pesar de esto, la estimación de su impacto varía en gran medida, dependiendo de los ejercicios de proyección: entre 0,4 y 2,3 puntos porcentuales del PIB durante los periodos comparados por la DGTPE. Varios supuestos sobre la salud de los adultos (por ejemplo, el envejecimiento saludable o no) o algunas diferencias metodológicas (por ejemplo, el tratamiento de los gastos relacionados con la muerte) podrían explicar estas diferencias.

Mecánicamente, el aumento de la población de edad avanzada llevaría a un aumento estructural de los gastos de salud, pero este hallazgo debe ser reevaluado, especialmente si el estado de salud de los ancianos está mejorando. El envejecimiento con pérdida de autonomía disminuye y, de forma saludable, tendrá un menor impacto en la evolución del gasto en salud.

El impacto que origina el envejecimiento en el aumento en los gastos en salud es del orden del 0,8\%, y podría estabilizarse entre 2015 y 2020. De acuerdo con las estimaciones del DGTPE basadas en las últimas proyecciones de población del Institut national de la statistique et des études économiques (INSEE), el impacto del envejecimiento sobre el gasto sanitario debe estar entre 0,5 y 2,5 puntos porcentuales del PIB en 2050. La cifra del medio punto del PIB se obtiene al suponer que la salud de los adultos mejorará paralelamente a lo largo de su vida. La cifra de 2,5 sería más realista si, al contrario, el cuidado de los ancianos se intensifica en el futuro. Si el perfil de gastos por edad se mantiene constante, el envejecimiento incrementaría 1,5 puntos porcentuales el gasto en salud del PIB para el año 2050. 


\section{La innovación técnica}

La búsqueda de innovación es otra tendencia que se espera mantener o fortalecer, y va en la dirección de un aumento sostenido del gasto. Varios campos ocultarían el potencial en este sentido: la lucha contra las enfermedades desconocidas, la medicina genética, nuevas formas de prevención y el diagnóstico precoz, la informatización de la medicina. El examen de los principales factores determinantes del gasto en salud sugiere que la innovación es un factor clave de crecimiento.

Si bien las evaluaciones disponibles de los futuros gastos en salud se basan generalmente en la reproducción de las tendencias del pasado, aparece una amplia gama de proyecciones: en 2050, la participación en el PIB del gasto en salud en Francia se incrementaría de 0 a más de 10 puntos.

A modo de ilustración, el punto medio de este rango (5 puntos del PIB) corresponde a un crecimiento anual del gasto en salud de, en promedio, un punto más que el PIB ( $5 \%$ del gasto sanitario frente al $4 \%$ del PIB, por ejemplo). Mejorar la calidad del servicio inducida por la innovación por sí sola es una explicación del aumento de precios.

Esta mejora tiende a optimizar la eficacia y la eficiencia deseada en términos de salud. Pero la forma de evaluar el precio de un cambio cualitativo correlacionado con un aumento en el rendimiento de los sistemas de atención de salud es también compleja de evaluar y plantea problemas de proyección.

También es necesario tener en cuenta el aumento del volumen de medicamentos que se consumen directamente vinculados a la innovación, porque nuevos productos son a menudo nuevas formas de los productos antiguos.

\section{Elasticidad}

Desde hace varias décadas, y en la mayoría de los países, se observa un aumento de la proporción de la riqueza nacional dedicada a la producción 
de servicios de salud. En Francia, el consumo de servicios de salud y productos médicos (Consommation de Soins et Biens Médicaux [CSBM]) representó el 3,4 \% del PIB en 1960; el 6,3\% en 1980; y el 8,7\% en 2007. Este crecimiento se refiere especialmente a la alta concentración de servicios de atención y a la previsión de los riesgos de enfermedades, lo que ha llevado prácticamente a todos los países de la OCDE a financiar los gastos en salud a través de un seguro público universal.

Si el peso en el PIB de la salud creció fuertemente durante los últimos cincuenta años, la continuidad de ese aumento en las próximas décadas no se puede dar por hecho;su magnitud es muy incierta, dadas las diversas variables sociales y macroeconómicas de las que depende.

A un lado de la balanza, los fenómenos que han fomentado el crecimiento del gasto en salud en el pasado ya no desempeñarán el mismo papel en el futuro, tales como la extensión de las coberturas de seguros, la creación y la inversión en estructuras hospitalarias.

La regulación y la gestión de políticas públicas también podrían ayudar a moderar los recursos en salud. Un aumento en el gasto de salud más rápido que el PIB es en el sistema actual la fuente de los problemas de financiación.

\section{Evaluación por la oferta de atención médica}

El gasto sanitario real no se decidirá solamente por el consumidor, sino también por el profesional de la salud. Indudablemente es la provisión de la atención la que ocupa el papel central en el volumen de la demanda de tratamientos. Los estudios sobre datos individuales documentan con suficiente claridad que los profesionales tienen la oportunidad de influir parcialmente en la demanda de salud, y una gran parte de los ellos dará lugar a una atención adicional.

Por su parte, la relación entre la densidad y el gasto médico es menos evidente. La densidad médica juega de manera muy diferente, 
dependiendo del método de remuneración del practicante: tendrá un efecto mayor cuando este es remunerado por cada tratamiento, un poco menos cuando le pagan por bloques de pacientes, y probablemente muy poco si es empleado.

La oferta es el determinante real de la demanda. Si todos los estudios intentan evaluar los gastos en función del consumo (demanda de atención), es conveniente tener en cuenta los estudios de evaluación cuyas variables principales se refieren a la oferta de tratamientos.

\section{Los modelos de proyección: las evaluaciones disponibles}

Parece muy difícil aislar los respectivos impactos de las variables socioeconómicas a partir de los trabajos existentes. Para desarrollar los escenarios de evolución, la elasticidad-ingreso se fijó en 1, dentro de los escenarios principales, lo que significa que los efectos de los ingresos no ejercen una presión adicional sobre el ratio de Gasto/PIB.

Las variables exógenas y supuestos que subyacen a las proyecciones

Se requiere una serie de datos externos para las proyecciones:

1. Las proyecciones de población se obtuvieron directamente de las fuentes nacionales de la Dirección de Empleo, Trabajo y Asuntos Sociales de la OCDE.

2. Las proyecciones de la tasa de participación de la población activa se basan en trabajos previos de la OCDE: se asume la influencia de las políticas vigentes en la tasa de actividad en las próximas décadas.

3. Se supone que el crecimiento de la productividad del trabajo converge linealmente, a partir de su tasa inicial (1995-2003) a una tasa anual de $1,75 \%$ en 2030 . 
Las proyecciones del PIB per cápita se calculan directamente usando las variables exógenas anteriores. Este marco sencillo está destinado a aislar tanto como sea posible el efecto del envejecimiento y otros factores demográficos sobre las proyecciones. La tabla 2 presenta el resumen de las proyecciones disponibles.

Tabla 2. Resumen de las proyecciones disponibles

\begin{tabular}{|c|c|c|c|c|c|c|c|c|}
\hline & 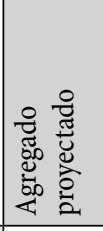 & 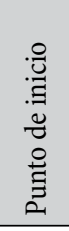 & 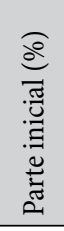 & 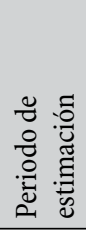 & 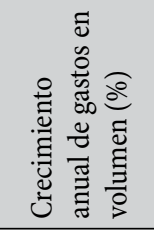 & 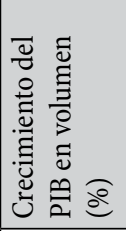 & \multicolumn{2}{|c|}{ 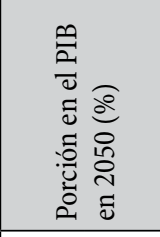 } \\
\hline $\begin{array}{l}\text { Proyección de } \\
\text { la OCDE }\end{array}$ & $\begin{array}{l}\text { Gastos } \\
\text { en } \\
\text { salud } \\
\text { pública }\end{array}$ & 2005 & 7,0 & $\begin{array}{l}1970- \\
2002 \\
1980- \\
2002\end{array}$ & $\begin{array}{c}\text { Entre } 2 \mathrm{y} \\
2,8\end{array}$ & $\begin{array}{c}1,8(1,5 \\
\text { por } \\
\text { cabeza })\end{array}$ & \multicolumn{2}{|c|}{$\begin{array}{c}\text { Entre } 7,3 \\
\text { y } 10,6\end{array}$} \\
\hline $\begin{array}{l}\text { Proyección } \\
\text { del Ageing } \\
\text { Working Group } \\
\text { de la Unión } \\
\text { Europea. }\end{array}$ & $\begin{array}{l}\text { Gastos } \\
\text { en } \\
\text { salud } \\
\text { pública }\end{array}$ & 2004 & 7,7 & $\begin{array}{l}1970- \\
2001\end{array}$ & 1,8 & 1,60 & 9,50 & \\
\hline $\begin{array}{l}\text { Extensión de } \\
\text { la proyección } \\
\text { realizada por el } \\
\text { senado }\end{array}$ & $\begin{array}{l}\text { Gastos } \\
\text { totales } \\
\text { en } \\
\text { salud }\end{array}$ & 2000 & 9,3 & $\begin{array}{l}1970- \\
2001\end{array}$ & \begin{tabular}{|c} 
2,5 (si el \\
crecimiento \\
del PIB es \\
de 1 ) \\
O \\
4,3 (si el \\
crecimiento \\
del PIB es \\
de 3 ) \\
\end{tabular} & $\begin{array}{c}2 \\
\text { hipótesis: } \\
1 \text { o } 3\end{array}$ & 17,40 & 19,40 \\
\hline $\begin{array}{l}\text { Proyección de } \\
\text { la DREES }\end{array}$ & $\begin{array}{l}\text { Gastos } \\
\text { totales } \\
\text { en } \\
\text { salud }\end{array}$ & 2004 & 10,4 & $\begin{array}{l}1971- \\
2002\end{array}$ & $\begin{array}{l}2,80 \\
3,70\end{array}$ & 2,00 & 14,90 & 22,30 \\
\hline
\end{tabular}

Fuente: OCDE (2012). 
En general, es posible establecer una evaluación cuantitativa de los factores de dinamismo futuro relativo al gasto en salud con relación al PIB. El poder del principal factor de crecimiento - renovación continua de la oferta que responde a la fuerte demanda potencial—, en combinación con la influencia del envejecimiento, lleva sin embargo a un gasto más dinámico que los ingresos (o que el PIB).

\section{B. Opiniones y perspectivas}

En su artículo, Cornilleau habla de las variables que según él pesan sobre el continuo aumento de los gastos en salud. En esta parte se presentan una serie de variables seleccionadas por otros autores y que sirven también para identificar los factores de aumento de los gastos.

\section{Las variables que permitan una evaluación de los costos de la salud}

Se pueden identificar los factores epidemiológicos como una variable que pesa sobre el gasto en salud. La morbilidad ${ }^{4}$ es un factor clave en el aumento del gasto en salud. Esto se debe, en gran parte, a enfermedades relacionadas con los estilos de vida, nuevas enfermedades —o reaparición de viejas enfermedades- 0 enfermedades crónicas.

El nivel de protección social es también una variable determinante, puesto que es un sistema que se financia en una tercera parte con contribuciones. El sistema de protección social para los franceses reduce las barreras financieras para acceder a la atención, pese al aumento en el volumen del consumo de bienes y servicios de salud. En la otra dirección, los seguros de salud complementarios influyen sobre el consumo de atención ambulatoria.

4 Número de personas que sufren de una enfermedad durante un periodo dado. 
La variable de residencia también es un factor que influye en el suministro de la atención. Aumenta el consumo de médicos en las ciudades, debido a la oferta, y disminuye en el campo.

El factor de la educación/categoría socio-profesional (CSP) tiene un modesto rol sobre el gasto en salud. Sin embargo, es importante tener en cuenta el grado de educación, ya que a mayor grado, mayor recurrencia a médicos especializados y a la atención dental. El efecto diploma induce a un alza en el consumo de bienes y productos médicos. La proliferación de centros de salud conlleva una densidad de la prestación de atención médica, que a su vez supone una mayor demanda de bienes y servicios de salud.

Respecto a la variable institucional, el crecimiento del gasto en atención de la salud se relaciona con los incentivos y las reglas establecidas por el marco institucional en el que se encuentran los asegurados y los profesionales de la salud. Como se ha visto, diferentes variables pueden explicar el aumento en el gasto en salud.

\section{Medidas que afectan la gestión del gasto en salud}

En el artículo, es lamentable ver que el autor no desarrolla políticas de gestión del gasto en salud. De hecho, desde un enfoque de prospectiva, habría sido interesante discutir las políticas que pueden tener un impacto en la reducción de los costos de salud. Sabiendo que hoy en día es imposible reducir algunos costos de salud, es importante gestionarlos.

Si se toma el trabajo de Dormont (2009), que descompone el crecimiento del gasto en salud francés en el periodo 1992-2000, se tienen los siguientes datos: el $58 \%$ se debe a cambios en la práctica; el $10 \%$, a cambios de enfermedad (y al mejoramiento de la salud); el $3 \%$, al aumento del tamaño de la población; y el $3 \%$, al envejecimiento. En conclusión a este aparte, en necesario enunciar algunas de las principales políticas de gestión:

- La meta nacional de los gastos de seguro de salud (ONDAM, definido por proyecto de ley de finanzas de la seguridad social). 
- La planificación sanitaria, que entre otras cosas establece objetivos cuantificados para la prestación de atenciones (OQOS).

- La financiación de hospitales (tarificación a la actividad).

- El mejoramiento de las vías de atención.

- La participación de los pacientes.

- La prevención y educación para la salud.

- El control de precios con las tarifas controladas.

- Medicamentos genéricos.

- El impedimento del reembolso de medicamentos.

- La mejora de las prácticas profesionales (HAS).

- La responsabilización de los prescriptores.

\section{Conclusión}

Como se ha visto a través del artículo de Gérard Cornilleau, desde el punto de vista de la economía de la salud, la salud no es un costo. La salud es un consumo especial, debido a la necesidad de los usuarios de garantizarse un seguro de salud (cáncer, infarto, etc.), y a su contribución al bienestar de la población.

Si bien en Francia la salud no tiene precio, sí tiene un costo, desde los años sesenta, el país ha experimentado un continuo aumento en el crecimiento del gasto en salud en el PIB. A pesar de los planes iniciales para el control de costos en 1975, dicha tendencia persiste. Desde entonces, el interés en la proyección de los gastos de salud y su costo siguen siendo temas críticos para el sistema de protección social. 
No existe un método único para determinar el costo de la salud. La dificultad para medirlo pasa por la inadecuada gestión del impacto de los determinantes del costo de la salud. Por un lado, los factores que sirven para extrapolar la evolución del gasto en salud se basan generalmente en series temporales de datos nacionales. Además, el Estado es el único propietario de la información y la fuente de su transmisión, lo cual pone en cuestionamiento la transparencia de los datos.

Es preciso el preguntarse por la pertinencia de los métodos para la determinación de los gastos de salud, que hasta hoy tiene en cuenta factores como la residencia, el efecto diploma o grado de educación y la práctica profesional.

Después de analizar todos estos elementos, queda un interrogante: ¿introducir una evaluación del impacto de las medidas de gestión del gasto en salud, así como una reforma de la financiación de la protección social, permitirá una mejor determinación del costo de la salud en el futuro?

\section{Referencias bibliográficas}

Asamblée National - République Française. (1946). Préambule de la Constitution du 27 octobre 1946. París: Asamblée National - République Française. .

Benamouzig, D., \& Launois, R. (1999). La maîtrise des dépenses de santé en France. París: REES France.

Cornilleau, G. (2009). Quel sera le coût de la santé demain? In Traité d'économie et de gestion de la santé (pp. 153-162). París: Presses de Sciences Po (P.F.N.S.P.).

Cutler, D. M., \& McClellan, M. (2001). Is technological change in medicine worth it? Health Affairs, 20(5), 11-29. Recuperado de http://doi.org/10.1377/ hlthaff.20.5.11

Direction de la Recherche des Études de l'Évaluation et des Statistiques (DREES). (2012). L'État de Santé de la Population en France. Suivi des Objectifs Annexés à la Loi de Santé Publique. Rapport 2011. DREES. Recuperado dehttp://www. sante.gouv.fr/IMG/pdf/Etat_sante-population_2011.pdf

Dormont, B. (2009). Les dépenses de santé: une augmentation salutaire? In Collection du CEPREMAP (p. 78). París: Rue d'Ulm. 
Dormont, B. (2011). Vieillissement et dépenses de santé. In La mondialisation de la recherche (1.st ed.). París: Collège de France.

Fargeon, V. (2009). Introduction à l'économie de la santé. Grenoble: Presses Universitaires de Grenoble. Recuperado dehttp://books.google.com.co/ books?id=8mbbQQAACAAJ

Horty, L., Quinet, A., \& Rupprecht, F. (1997). Les evolutions de la demande des menages: facteurs economiques. París: INSEE, Division Conditions de vie des ménages.

Kinnon, C., Velazquez, G., \& Flori, Y. A. (1994). Economie de la santé. Choix de textes de l'OMS (1.st ed.). Ginebra: OMS.

L'Assurance Maladie. (2008). Histoire de l'Assurance Maladie. Recuperado de http:// www.ameli.fr/l-assurance-maladie/connaitre-l-assurance-maladie/missionset-organisation/la-securite-sociale/histoire-de-1-8217-assurance-maladie.php

Murillo, C., Piatecki, C., \& Saez, M. (1993). Health care expenditure and income in Europe. Health Economics, 2(2), 127-138. Recuperado de http://doi. org/10.1002/hec.4730020206

Newhouse, J. P. (1977). Medical-Care Expenditure: A Cross-National Survey. The Journal of Human Resources, 12(1), 115-125. Recuperado de http://doi. org/10.2307/145602

OCDE. (2012). Organización de Cooperación y de Desarrollo Económico. Recuperado dehttp://stats.oecd.org/index.aspx?DataSetCode=HEALTH_STAT\&lang=fr

Phelps, C. (1995). Les fondements de l'économie de la santé (1.st ed.). París: Publi-Union. 